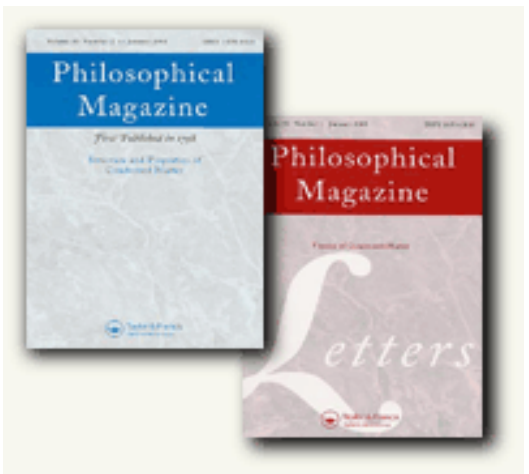

\title{
Modeling size effects using 3D density-based dislocation dynamics
}

\begin{tabular}{|r|l|}
\hline Journal: & Philosophical Magazine \& Philosophical Magazine Letters \\
\hline Manuscript ID: & TPHM-06-Jun-0215.R1 \\
\hline Journal Selection: & Philosophical Magazine \\
\hline Date Submitted by the & 28-Jul-2006 \\
\hline Complete List of Authors: & $\begin{array}{l}\text { Zaiser, Michael; The University of Edinburgh, King's Buildings, } \\
\text { Sanderson Building, Center for Materials Science and Engineering } \\
\text { Nikitas, Nikos; The University of Edinburgh } \\
\text { Hochrainer, Thomas; University of Karlsruhe } \\
\text { Aifantis, Elias; University of Thessaloniki }\end{array}$ \\
\hline Keywords: & constitutive equations, dislocation mechanics, plastic deformation \\
\hline Keywords (user supplied): & \\
\hline $\begin{array}{l}\text { Note: The following files were submitted by the author for peer review, but cannot be converted } \\
\text { to PDF. You must view these files (e.g. movies) online. }\end{array}$ \\
\hline PhilMagProduction.tex
\end{tabular}

\section{S) ScholaroNE \\ Manuscript Central}




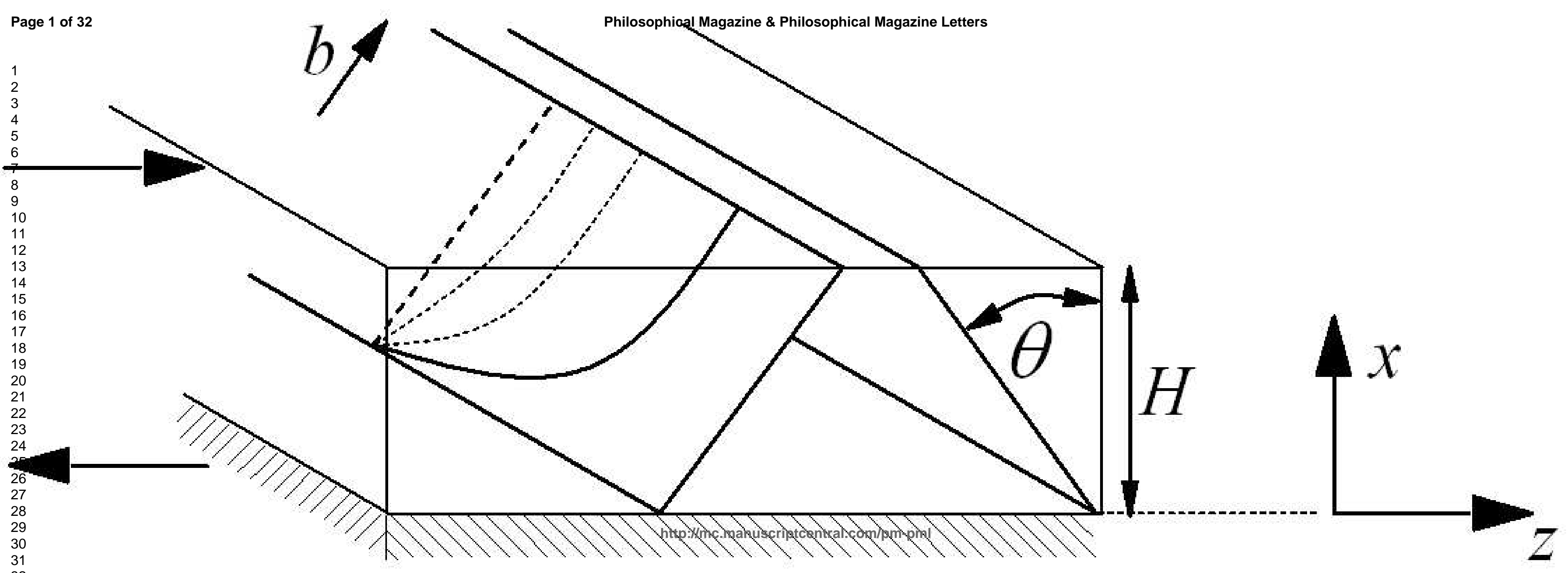




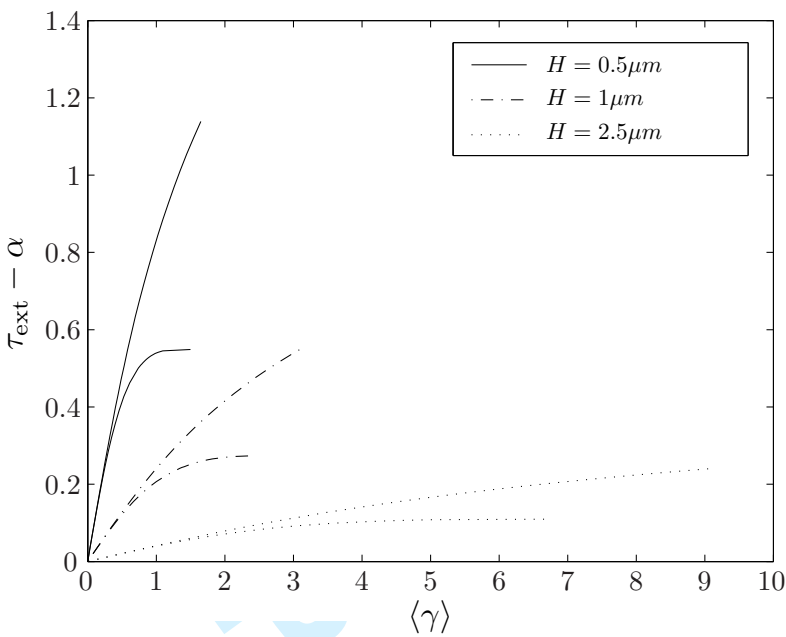



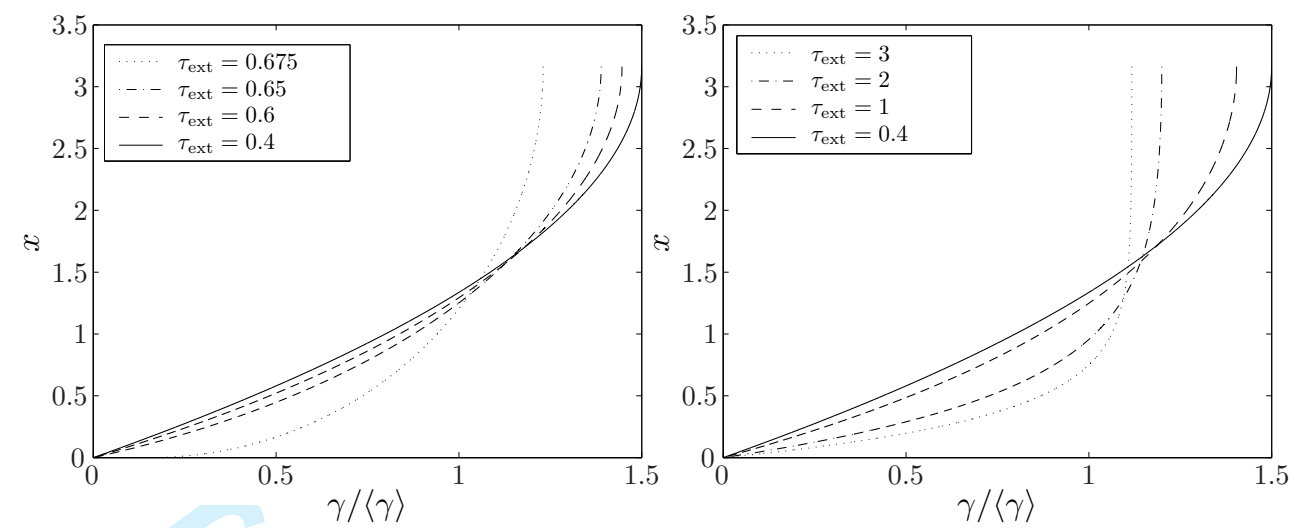


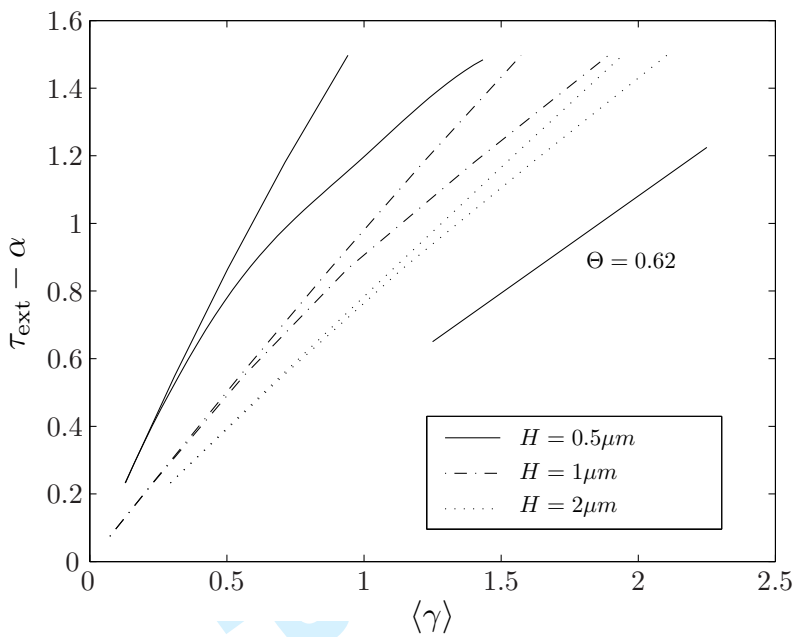



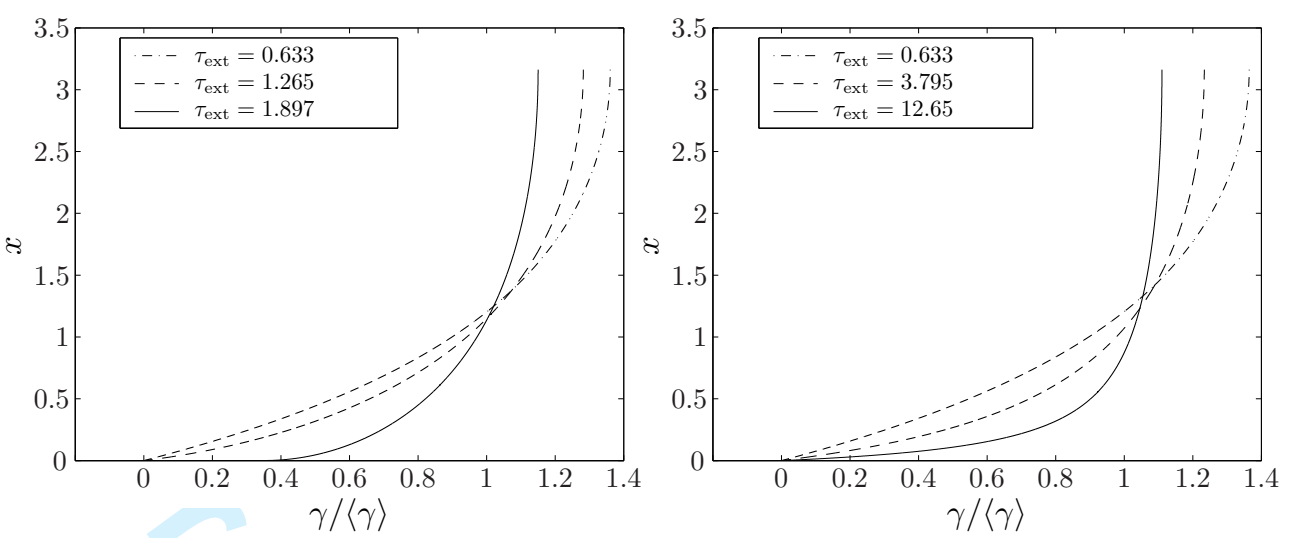


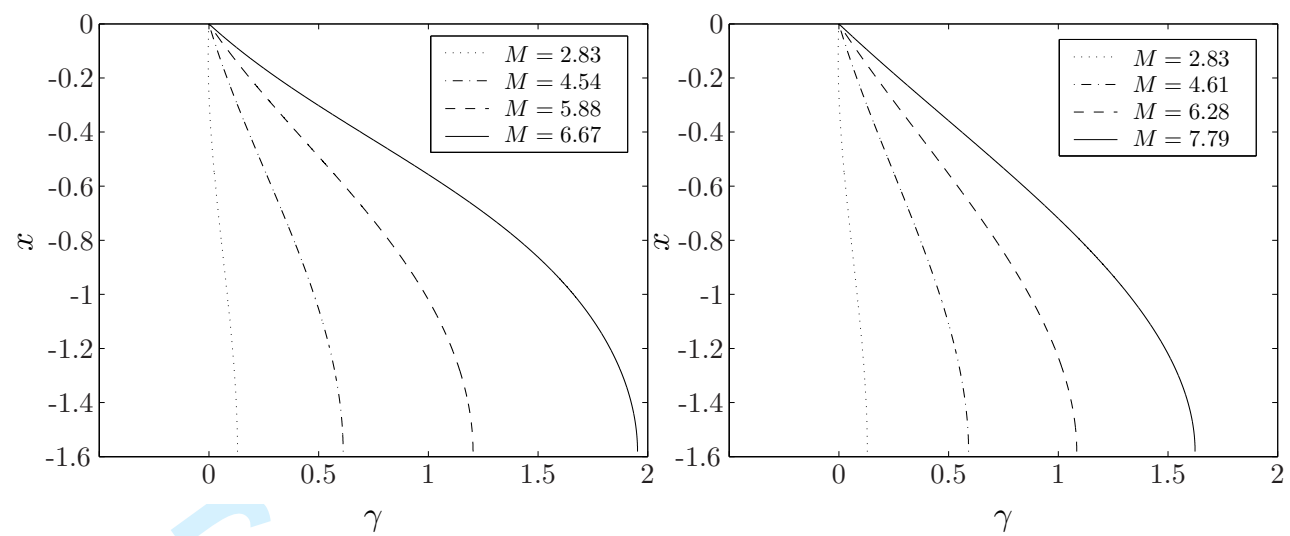


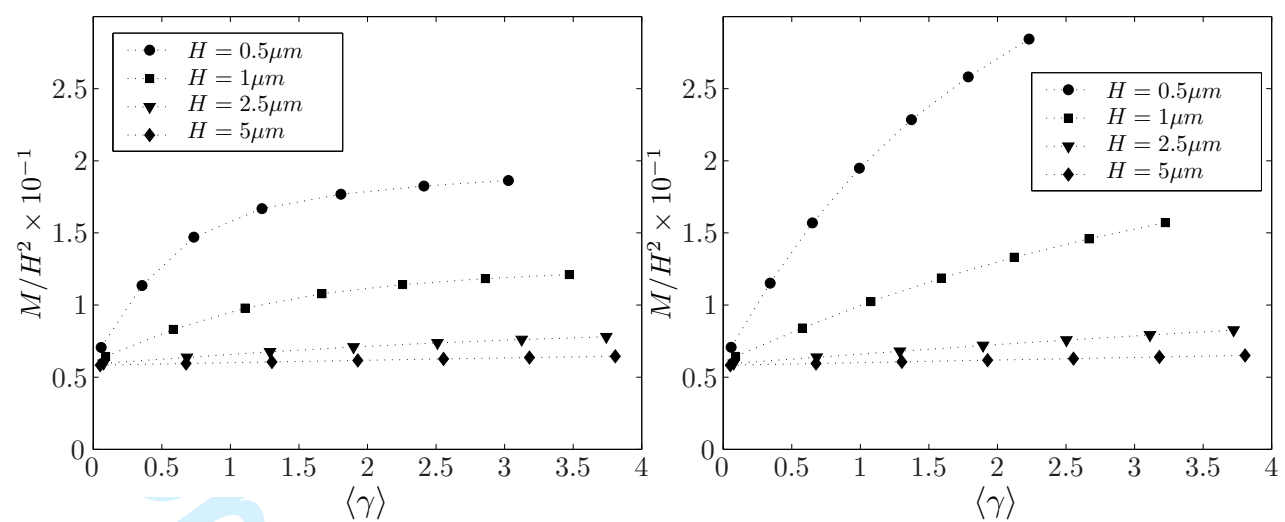




\title{
Modeling size effects using 3D density-based dislocation dynamics
}

\author{
July 28,2006 \\ Michael Zaiser ${ }^{1}$, Nikos Nikitas ${ }^{1,2}$, Thomas Hochrainer ${ }^{3}$ \\ AND E.C. Aifantis ${ }^{2}$ \\ 1 The University of Edinburgh, Institute for Materials and Processes, \\ The King's Buildings, Sanderson Building, Edinburgh EH9 3JL, UK \\ ${ }^{2}$ Aristotle University of Thessaloniki, Polytechnic School, 54006 Thessaloniki, \\ Greece \\ ${ }^{3}$ Universität Karlsruhe (TH), Institut für Zuverlässigkeit \\ von Bauteilen und Systemen, Kaiserstr. 12, 76131 Karlsruhe, Germany
}

\begin{abstract}
We use density-based continuity equations to model strain patterns and size effects in confined plastic flow, namely, shearing of thin films, and micro-bending. To this end, we use a representation in terms of coupled equations for the densities of screw and edge components. We show how these equations derive from a more general formulation in a higher-dimensional configuration space, and discuss relations with other density-based approaches proposed in the past. The new element here is the incorporation into previous continuum formulations of geometrical features and interactions of dislocation lines that cannot be neglected or 'averaged out' within a three-dimensional setting of plasticity at the micron and nano scales.
\end{abstract}

\section{Introduction}

Plastic deformation of crystals results from the motion of interacting dislocation lines. As dislocations move and interact under the action of stresses applied to the deforming body, they may form patterns of intriguing complexity. Such 'dislocation patterning' [1,2] phenomena appear over a wide spectrum of space and time scales in the form of dipoles or multipoles, walls or pileups, cells or subcells, stationary or travelling strain bands. Thus, the pattern of discrete dislocation lines in the deforming crystal, and the associated pattern of internal stresses, exhibit various internal length scales ranging from the 'elementary' length scale defined by the dislocation spacing over the scale of dislocation cells and sub-grains to the macroscopic scale of deformation bands.

Accordingly, understanding the spatial organization of dislocation systems is of key importance not only for capturing the internal stress state of a ma- 
terial and the associated material behavior (hardening/softening), but also for analysing the interplay of internal and external length scales as it manifests itself in the form of size effects in plasticity. Two main approaches have been pursued so far: Discrete Dislocation Dynamics (DDD) and Continuum Density-based Dislocation Dynamics (CDDD).

(i) Discrete Dislocation Dynamics (DDD) traces the evolution of the dislocation systems on a 'microscopic' scale where discrete dislocations can be resolved - either by investigating the motion of discrete lines, which may be discretised into segments $[3,4]$ or treated as continuous lines $[5,6,7]$ or by tracing the evolution of strain $[8,9]$ or dislocation fields $[10,11]$ on a 'microscopic' scale. Irrespective of the formulation used, this approach has the advantage, but also the drawback, that the complete information about the microscopic stress and deformation state is retained. This has obvious advantages in terms of conceptual simplicity, and equally obvious disadvantages in terms of computational cost. Indeed, because of the huge computational cost there has been, for instance, no large-scale discrete simulation of dislocation cell patterning despite 15 years of dedicated effort. In addition, simulation approaches based on discrete lines fit badly into the traditional conceptual framework of continuum plasticity and to 'interface' them with averaged continuum descriptions of the stress and strain states is a formidable problem of multiscale materials modeling.

(ii) Continuum Density-based Dislocation Dynamics (CDDD) describes the evolution of the dislocation microstructure in terms of continuous field variables for which evolution equations are formulated. Historically this approach has three roots: (a) simple internal-variable models as first advocated by Gilman and later elaborated by Kocks, Mecking, and their school (see e.g. [12, 13]). In these, the microstructure is described in terms of internal variables ('dislocation densities') which evolve with strain but do not possess any spatial degrees of freedom (i.e., they do not undergo transport); (b) quasi-thermodynamic models which derive the transport dislocations and their spatial organization into cells and clusters from internal energy minimization $[14,15]$; (c) the complex and geometrically rigorous Kröner-type theories of continuous distributions of dislocations $[16,17,18,19]$ (for overwiews, see $[20,21]$ ) which, however, were never truly elaborated into a theory of the dynamic evolution of dislocation systems except on the level of discrete dislocation lines [10].

Dislocation transport was introduced into CDDD by Aifantis and co-workers $[22,23,24,25,26,27,28]$, and in a slightly different manner by Kratochvil [29] (for a recent elaboration of this approach see [30]). The primary purpose of introducing transport terms was to model dislocation patterning phenomena, in particular the emergence of periodically ordered persistent slip band and matrix patterns in cyclic deformation. Drawing on phenomenological analogies with pattern formation in other reaction-diffusion systems, evolution equations of the reaction-diffusion and reaction-diffusion-transport type were formulated for different dislocation 'species' or 'families' distinguished by their geometrical and dynamical properties (positive vs. negative, mobile vs. immobile dislocations, moving dislocations vs. immobile dipoles). Internal length scales appear in this type of theories in terms of diffusionlike transport terms which have been linked to the cyclic mean free path of dislocations [31] or, more generally, to their characteristic transport length before collisions (dislocation reactions). This approach was later also applied to understand the spatial organization of deformation on macroscopic scales during plastic instabilities where multiplica- 
tion and diffusionlike propagation of dislocations may lead to the emergence of propagating deformation bands [32, 33, 34].

A topic which has been of particular interest in recent years concerns size effects in plasticity and their modelling using continuum constitutive equations with internal length scales. Modelling has been mainly done using phenomenological models which incorporate strain gradient terms into constitutive laws for continuum plasticity $[35,36,37,38,39,40]$ but the relation with dislocation behavior has not generally been made, even though arguments invoking geometrically necessary dislocations are commonplace (e.g. [36, 37], for an overview see [41]). A critical discussion of these arguments has been given by Zaiser and Aifantis [42], who also illustrated the application of phenomenological reactiontransport equations for dislocation densities to size effects in microbending.

Recently, various attempts have been made to go beyond phenomenological evolution equations and to formulate the evolution of dislocation densities within a more rigorous mathematical framework. In particular, Groma, Zaiser and co-workers have addressed the evolution of systems of parallel straight dislocations in terms of concepts borrowed from the statistical mechanics of interacting many-particle systems, which allow to derive equations of evolution for dislocation density fields from the dynamics of discrete dislocations through systematic averaging procedures.

For systems of straight parallel dislocation lines, Groma, Zaiser and coworkers have developed a formalism to represent both the kinematics and interactions of dislocations in a continuum framework [43]. Averaging the discrete equations of motion leads to a hierarchy of equations for many-dislocation densities which is truncated at second order, i.e., the interaction of a dislocation with others in its vicinity is represented in terms of pair correlation functions. Owing to the short-range nature of these correlations [44,45], the interaction can be approximately described in terms of a density dependent flow stress plus a strain-gradient dependent 'back stress' term, whereas the interactions between distant dislocations are appropriately characterized in terms of a longrange stress field which is a functional of the dislocation density pattern. This approach has been tested against numerous benchmark problems and, in general, yields excellent agreement between 2D discrete simulations and continuum calculations both $[46,47]$.

In $3 \mathrm{D}$, the problem is more complicated since the kinematic description of systems of moving lines (as opposed to point particles in 2D) is not straightforward. Different kinematic formulations have been proposed in the literature $[48,49]$ while a general analysis which allows to recover these formulations as special cases has been provided by Hochrainer [50]. In the present paper, we first formulate a density-based theory of the kinematics, dynamics and interactions of systems of directed and connected dislocation segments moving on a single slip system. We then discuss several examples where this is applied to size effects in constrained plastic flow (channel slip, shearing of thin films) and in microbending. Particular attention is devoted to assessing the respective influences of dislocation self-interactions (line tension) and interactions between different dislocations on the size-dependent yielding and hardening behavior. We also use these examples to emphasize the importance of initial and boundary conditions for the evolution of the dislocation system, and their possibly crucial influence on the plastic response of a material. It is noted that such an approach is more rigorous and reliable over other dislocation or strain gradient 
models for predicting size effects in small metal volumes such as those involved in multilayer films and micro/nanoindentation where discrete dislocation features should be effectively incorporated into continuum formulations.

\section{Internal stresses, dislocation kinematics and dynamics}

\section{$2.1 \quad$ Kinematics and dynamics}

In the following we use as a starting point the kinematic framework developed by Hochrainer $[50,51,52]$, i.e., we use a dislocation density measure $\rho$ which is defined on a configuration space which consists of the positions and orientations of dislocation segments, and treat the dislocation line curvature $k$ as an independent field variable. We specify our formulation to dislocations moving by glide on a given slip system, such that the dislocation orientation can be expressed in terms of a scalar variable $\phi$ which we define as the angle between the dislocation line direction and the Burgers vector. (Situations with dislocations moving on multiple slip systems can be treated straightforwardly by defining separate measures $\rho^{i}$ for each slip system $i$; in the following we drop the subscript $i$ for the simplicity of presentation.)

For a given orientation, we define a set of unit vectors $\boldsymbol{n}$ in the direction of the glide plane normal, $\boldsymbol{e}_{\mathrm{t}}(\phi)$ in the direction tangential to the dislocation line, and $\boldsymbol{e}_{\mathrm{g}}(\phi)=\boldsymbol{n} \times \boldsymbol{e}_{\mathrm{t}}(\phi)$ in the dislocation glide direction. Assuming that dislocations move by glide only, the evolution of the density measure $\rho$ is then given by the continuity equation (cf. [51], this volume)

$$
\partial_{t} \rho=-\partial_{\phi}\left[\rho v_{\phi}\right]-\nabla \cdot[\rho \boldsymbol{v}]+\rho v k
$$

Here, $\boldsymbol{v}=\boldsymbol{e}_{\mathrm{g}} v$ is the vectorial and $v$ the pseudo-scalar velocity of the dislocation segments, and the rotation velocity of the dislocation lines is given by

$$
v_{\phi}=\boldsymbol{e}_{\mathrm{t}} \cdot \nabla v+k \partial_{\phi}[v] .
$$

It is noted that the conservation law given by Eq.(1) is a generalization of previously formulated continuity equations for straight parallel dislocations (e.g., $[2,43])$. The curvature-dependent term contained in the above continuity equation accounts for the expansion or shrinkage of dislocation loops, and in a implicit manner for dislocation reaction (generation/annihilation) terms as they have been introduced e.g. by Aifantis $[2,31,38]$. (Note that, in a threedimensional network of flexible dislocations, reactions necessarily occur through the formation, motion and dissolution of nodes, i.e. of singularities in the curvature field.)

The dislocation curvature is, in the kinetic theory of Hochrainer, understood as an additional field variable. The theory is exact if all dislocations of a given orientation in a given volume element have the same curvature [51]. The local values of the curvature field pertaining to a given orientation then obey the differential equation

$$
\partial_{t} k=-k^{2} v+k\left(\boldsymbol{e}_{\mathrm{g}} \cdot \nabla v\right)-\boldsymbol{e}_{\mathrm{t}} \cdot \nabla\left[v_{\phi}\right]-k \partial_{\phi}\left[v_{\phi}\right]-\boldsymbol{v} \cdot \nabla[k]-v_{\phi} \partial_{\phi}[k] .
$$


The equations for the curvature field may be solved alongside with those for the dislocation density measure. Alternatively, in the present investigation we use later on a line tension approximation to evaluate the dislocation behaviour under quasi-static conditions. In this approximation, the curvature ceases to be an independent field and simply becomes a function of the shear stress acting on the dislocation, and thereby a functional of the dislocation density measure.

The plastic strain rate can be recovered from the dislocation density measure as

$$
\partial_{t} \gamma(\boldsymbol{r})=\int(\rho b v) \mathrm{d} \phi,
$$

where $b$ is the modulus of the Burgers vector and the integral is extended over all orientations $\phi \in[0,2 \pi]$. This relation may be integrated alongside with the evolution of the dislocation density measure to yield the plastic strain field at a given moment in time.

To pass from dislocation kinematics to dynamics, we have to specify the dislocation velocity $v$. We neglect dislocation inertia and assume that the dislocation velocity is controlled by drag forces. In this case, the velocity becomes a function of the local stress $\tau(\boldsymbol{r})$ acting on a dislocation at $\boldsymbol{r}$,

$$
\frac{v}{B(\phi)}=\tau(\boldsymbol{r}) b
$$

where $B$ is the (in general direction-dependent) dislocation mobility. The key problem is now how to evaluate the stress $\tau(\boldsymbol{r})$ which, in order to obtain a closed theory of plastic flow, must be expressed as a functional of the plastic strain and/or the dislocation density measure.

\section{$2.2 \quad$ Internal stresses}

As already mentioned, by integrating Eq.(4), we can obtain the plastic strain field at a given moment in time. It is then a standard problem of continuum mechanics to determine the corresponding stress field for a given set of boundary conditions. In the following we use a terminology where we split this stress field into a field $\tau_{\text {ext }}$ ('external stress') that is due to the tractions applied from outside to the deforming body, and a mesoscopic internal-stress field $\tau_{\mathrm{mf}}$ which arises from the in general inhomogeneous strain field $\gamma(\boldsymbol{r})^{1}$. In a small-strain approximation (and only then) the two contributions are additive because of the linearity of the problem. In an infinite crystal, the mesoscopic stress $\tau_{\mathrm{mf}}$ can be expressed as a functional of the dislocation density pattern via

$$
\tau_{\mathrm{mf}}(\boldsymbol{r})=\int_{V} \rho\left(\boldsymbol{r}^{\prime}, \phi^{\prime}\right) \tau_{\mathrm{seg}}\left(\boldsymbol{r}-\boldsymbol{r}^{\prime}, \phi^{\prime}\right) \mathrm{d} \boldsymbol{r}^{\prime} \mathrm{d} \phi^{\prime}
$$

where $\tau_{\text {seg }}$ is the shear stress created at $\boldsymbol{r}$ by a segment of orientation $\phi^{\prime}$ located at $\boldsymbol{r}^{\prime}$. Explicit expressions for segment stresses have been given by Devincre and Condat [3]. We note, however, that, instead of evaluating the expression given by Eq.(6) it is often more convenient to solve the elastic problem directly;

\footnotetext{
${ }^{1}$ The notion of a 'mesoscopic' internal-stress field is motivated by the fact that this stress field is defined on the same scale as the dislocation density measure $\rho$ and the plastic strain $\gamma$. If $\rho$ and $\gamma$ are considered as smooth averages over volume elements containing many dislocations, the same holds for $\tau_{\mathrm{mf}}$.
} 
in cases where surface boundary conditions are imposed this may indeed be the only feasible method of solution, and such a scheme is also adopted in the subsequent examples.

The mesoscopic stress field, Eq.(6), varies on the same length scale as the dislocation density pattern described by the dislocation density measure $\rho$. In principle, $\rho$ can be considered with a spatial resolution sufficient such that individual dislocations can be resolved; in this case the complete information about the internal stresses is contained in Eq.(6), and our theory is just a complicated way of expressing the evolution of a discrete dislocation system. However, the main point of a density-based description is to describe dislocation dynamics on a mesoscopic scale where the 'elementary size' of a volume element may be above the mean dislocation spacing. In this case, any fluctuations of the stress field on the scale of individual dislocations are averaged out. A straightforward proposition might be to describe, within the framework of such a mesoscopic theory, the local stress state in terms of $\tau(\boldsymbol{r})=\tau_{\mathrm{ext}}+\tau_{\mathrm{mf}}$ and to evaluate $\tau_{\mathrm{mf}}$ - which in this case has the character of a mean stress field - from the (mesoscopically averaged) dislocation density measure, or the (also mesoscopically averaged) strain field. This amounts to a mean-field dislocation theory of plasticity as discusssed by Hochrainer et. al. [51] (this volume), or (in different versions) by other authors in the past (e.g. Aifantis [32]).

It is, however, emphasized that such a mean-field theory, which from a statistical viewpoint corresponds to neglecting any correlations between dislocation segments and/or dislocation lines, in general does not provide a full description of the stresses acting locally on the dislocations. This can be seen immediately by considering an infinite body deforming (on mesoscopic scales) in homogeneous simple shear. In this case, the mesoscopic shear strain is a constant, and accordingly the mesoscopic stress $\tau_{\mathrm{mf}}$ is zero. Since in a mean-field theory $\tau_{\mathrm{mf}}$ is the only term which accounts for dislocation interactions, such a theory predicts for mesoscopically homogeneous dislocation arrangements that, irrespective of the dislocation arrangement within the elementary volume, the flow stress is zero unless obstacles other than dislocations are introduced in some way into the stress-velocity law. It can be seen from dislocation dynamics simulations but also from experiment (consider hardening Stage I of pure fcc metals) that this assertion is incorrect.

A straightforward extension of the mean-field approach, which is particularly well-adapted to the formulation used in the present paper, is to include into the theory stresses associated with the large-scale curvature of the dislocations. In a line-tension approximation, such stresses can be expressed as $\tau_{\mathrm{lt}}=T k / b$ where $T \approx G b^{2}$ is the dislocation line tension. Note, however, that $k$ in the present formulation is considered as a mesoscopic field, and therefore $\tau_{1 \mathrm{t}}$ does not account for stresses due to fluctuations of the lineshape on the 'microscopic' scale below the average dislocation spacing. Such stresses, which arise from bowing out of dislocations between junctions and other localized obstacles, form in a mesoscopic description part of the flow stress. This is discussed in the next paragraph.

In general, dislocations during plastic flow arrange themselves in such a way as to mutually obstruct their motion. These 'jammed' dislocation configurations [53] may consist of dislocation dipoles and multipoles, or of dislocation junctions and locks. In either case, such configurations have an extension of one or at maximum a few dislocation spacings, i.e. they pertain to a 'microscopic' 
length scale below the averaging scale of a mesoscopic theory. Formally, such configurations and the associated 'microscopic' stresses can be included into a mesoscopic theory by considering dislocation-dislocation correlation functions. This has been discussed for 2D dislocation systems (systems of parallel edge dislocations) by Zaiser, Groma and co-workers [44, 43], and a similar procedure for $3 \mathrm{D}$ dislocation systems was outlined in $[52,54]$. The basic idea is that dislocation pair correlations are short-ranged with a range of the order of one dislocation spacing. Therefore, the dislocation interactions which are associated with them can, on a mesoscopic scale, be expressed as local functions of the averaged dislocation densities and their gradients (local density approximation). The short-range nature of the correlation functions allows for a systematic expansion into dislocation density gradients of increasing order. The assertion that dislocation pair correlations are short-ranged has recently been confirmed for systems of straight parallel dislocations in a study by Groma et. al. [45] (this volume). They derive an analytical expression for the pair correlation function and demonstrate quantitative agreement with discrete dislocation dynamics simulations.

In the following we shall not analyse dislocation-dislocation correlations in detail (for such an analysis, see the papers of Groma and co-workers [45] and of El-Azab and co-workers [55] in the present volume). Rather, we adopt a phenomenological approach where we describe the 'microscopic' stress state in terms of a dislocation-density dependent friction stress $\tau_{\mathrm{f}}$ and a 'back stress' $\tau_{\mathrm{b}}$ which depends on the dislocation density gradient (see also the introduction of similar expressions in [56]). These are assumed in the form

$$
\tau_{\mathrm{f}}=\alpha G b \sqrt{\rho_{\mathrm{t}}}, \tau_{\mathrm{b}}=\frac{D G b}{\rho_{\mathrm{t}}} \int\left(\boldsymbol{e}_{\mathrm{g}} \cdot \nabla \rho\right) \mathrm{d} \phi .
$$

Here $\rho_{\mathrm{t}}=\int \rho \mathrm{d} \phi$ denotes the total dislocation density in a given point and $G$ is the shear modulus of the material. The constants $\alpha$ and $D$ may in general depend on the line orientation $\phi$ and/or on the 'composition' of the dislocation arrangement (the distribution of dislocations over the different directions). However, the basic structure of the 'flow stress' and 'friction stress' terms is dictated by scaling properties as discussed in [44, 43] for two- and in [52, 54] for three-dimensional dislocation systems. In particular, for a $2 \mathrm{D}$ dislocation arrangement consisting of straight parallel edge dislocations these terms reduce to expressions derived by Groma and co-workers using both statistical averaging techniques [43] and extremal principles ([45], this volume). In passing, we note that the 'flow stress' expression is simply Taylor's well-established relation for the dissipative friction stress that has to be overcome when deforming a dislocation arrangement, whereas the 'back stress' is a 3D generalization of the 2D 'pile-up stress' derived by Groma and Zaiser, which has been proven in numerous studies of size-dependent plasticity to yield a correct continuum representation of short-range dislocation repulsion in $2 \mathrm{D}$ dislocation dynamics and the associated size effects.

In the following we use Eq. (7) with isotropic (direction-independent) coefficients $\alpha$ and $D$ to describe the 'microscopic' stress state and combine this with a continuum approach to calculate the external and mesoscopic internal stresses. Before applying the formalism to concrete examples, however, it is convenient to re-formulate the kinetic equations in a form that is suitable for an analytic treatment of the simple example problems we are going to discuss. 


\subsection{Edge-Screw Representation}

A practical hindrance to numerical implementation of the continuum dislocation dynamics formulated above is that a reasonable resolution in angular space requires a large number of dislocation fields $\rho\left(\boldsymbol{r}, \phi_{i}\right)$. Since this obviously increases the numerical cost, one may ask whether it is possible to use only a coarse discretization, such as the edge-screw model proposed by Arsenlis et. al. [57]. However, a 'naive' edge-screw approach which allows for only 4 values of $\phi(\phi \in[0, \pi / 2, \pi, 3 \pi / 2])$ is problematic since line-tension effects and dislocation multiplication are badly represented (one effectively deals with rectangular dislocation loops). None of the subsequent examples can be adequately treated in this too-simplistic approximation.

We therefore use a different approach where we start out from the kinematic formulation outlined in Section 2.1, but then represent the dislocation orientation in terms of the edge and screw components of mixed dislocation segments. We first consider the density measure $\rho$ on a 'microscopic' scale where individual dislocation lines can be resolved, and associate it with the screw and edge segment densities $R_{\mathrm{s}}$ and $R_{\mathrm{e}}$. On the microscopic scale, the screw, edge, and total densities fulfil the geometrical relation $\rho^{2}=R_{\mathrm{s}}^{2}+R_{\mathrm{e}}^{2}$, and the local dislocation orientation is $\tan \phi=R_{\mathrm{e}} / R_{\mathrm{s}}$. Assuming that the Burgers vector points in the $x$ direction and considering a direction-independent mobility $B$, we obtain from Eqs. (1),(2) the following equations of evolution for the screw and edge densities (for details of the derivation see Appendix):

$$
\begin{aligned}
& \partial_{t} R_{\mathrm{e}}=\left[-\frac{R_{\mathrm{e}}^{2}}{\rho^{2}} \partial_{x}(\rho v)+\frac{R_{\mathrm{e}} R_{\mathrm{s}}}{\rho^{2}} \partial_{y}(\rho v)\right]+R_{\mathrm{s}}\left[\frac{R_{\mathrm{s}}}{\rho} \partial_{x} v+\frac{R_{\mathrm{e}}}{\rho} \partial_{y} v\right]+R_{\mathrm{e}} v k \\
& \partial_{t} R_{\mathrm{s}}=\left[-\frac{R_{\mathrm{e}} R_{\mathrm{s}}}{\rho^{2}} \partial_{x}(\rho v)+\frac{R_{\mathrm{s}}^{2}}{\rho^{2}} \partial_{y}(\rho v)\right]-R_{\mathrm{e}}\left[\frac{R_{\mathrm{s}}}{\rho} \partial_{x} v+\frac{R_{\mathrm{e}}}{\rho} \partial_{y} v\right]+R_{\mathrm{s}} v k
\end{aligned}
$$

It is important to note that in this model edge and screw segments move together in the glide direction determined by the local 'composition' of the dislocation line and not separately by the 'edge' and 'screw' components.

In the following we are interested in a statistical description of the dislocation system. We therefore proceed to average these equations. We introduce the notations $\kappa_{\mathrm{s}}:=\left\langle R_{\mathrm{s}}\right\rangle, \kappa_{\mathrm{e}}:=\left\langle R_{\mathrm{e}}\right\rangle, \rho:=\langle\rho\rangle, \rho_{\mathrm{s}}^{2}:=\left\langle R_{\mathrm{s}}^{2}\right\rangle, \rho_{\mathrm{e}}^{2}:=\left\langle R_{\mathrm{e}}^{2}\right\rangle$ and $\rho_{\mathrm{se}}^{2}:=$ $\left\langle R_{\mathrm{s}} R_{\mathrm{e}}\right\rangle$, where the averages are understood as both spatial and directional. We obtain

$$
\begin{gathered}
\partial_{t} \kappa_{\mathrm{e}}=\left[-\frac{\rho_{\mathrm{e}}^{2}}{\rho^{2}} \partial_{x}(\rho v)+\frac{\rho_{\mathrm{se}}^{2}}{\rho^{2}} \partial_{y}(\rho v)\right]+\rho\left[\frac{\rho_{\mathrm{s}}^{2}}{\rho^{2}} \partial_{x} v+\frac{\rho_{\mathrm{se}}^{2}}{\rho^{2}} \partial_{y} v\right]+\kappa_{\mathrm{e}} v k, \\
\partial_{t} \kappa_{\mathrm{s}}=\left[-\frac{\rho_{\mathrm{se}}^{2}}{\rho^{2}} \partial_{x}(\rho v)+\frac{\rho_{\mathrm{s}}^{2}}{\rho^{2}} \partial_{y}(\rho v)\right]-\rho\left[\frac{\rho_{\mathrm{se}}^{2}}{\rho^{2}} \partial_{x} v+\frac{\rho_{\mathrm{e}}^{2}}{\rho^{2}} \partial_{y} v\right]+\kappa_{\mathrm{s}} v k
\end{gathered}
$$

for the average sign densities. In deriving these equations we have used the relations $\left\langle R_{\mathrm{e}}^{2} / \rho\right\rangle=\left\langle R_{\mathrm{e}}^{2}\right\rangle\langle\rho\rangle /\left\langle\rho^{2}\right\rangle$ and similarly for $R_{\mathrm{s}}$, which are proven in the Appendix. As we assumed that the dislocation velocity is independent of the segment orientation, there is no correlation between $\left(R_{\mathrm{e}} / \rho\right),\left(R_{\mathrm{S}} / \rho\right),\left(R_{\mathrm{e}}^{2} / \rho^{2}\right)$, or $\left(R_{\mathrm{s}}^{2} / \rho^{2}\right)$, and $v$. We note that, for a system of parallel edge dislocations where $R_{\mathrm{s}}=0$ and hence $\rho_{\mathrm{se}}=\rho_{\mathrm{s}}^{2}=0, \rho_{\mathrm{e}}^{2}=\rho^{2}$, Eq.10 reduces to the simple relation

$$
\partial_{t} \kappa_{\mathrm{e}}=-\partial_{x}(\rho v)
$$


which is one of the two equations describing the kinetics of such a system as discussed by Groma et. al. [43]. Comparison with that work also demonstrates that a closed description requires a second equation for the total density $\rho$ (or, equivalently, the density square $\rho^{2}$ ). In the edge-screw case, we need in general three additional equations as Eqs. (11) and (10) contain the product densities $\rho_{\mathrm{s}}^{2}, \rho_{\mathrm{e}}^{2}$ and $\rho_{\mathrm{se}}^{2}$. For these, evolution equations can be obtained by averaging the evolution of the products $R_{\mathrm{s}}^{2}, R_{\mathrm{e}}^{2}$, and $R_{\mathrm{s}} R_{\mathrm{e}}$. In the examples discussed in the present paper, however, it is in fact sufficient to consider Eqs. (10) only, as will become evident in the following.

\section{Examples}

In the following we consider some examples of plane-strain deformation to thin films under different boundary conditions, viz, the shearing of a thin film tethered to a non-deformable substrate and containing either one single or two symmetrically inclined slip systems, and the bending of a free-standing thin strip containing two symmetrically inclined slip systems. In either case, we assume that, as an initial condition, equal densities $\rho_{0} / 2$ of screw dislocations of both signs but no edge segments are present. Owing to the plane-strain geometry, the density of screw segments is conserved for this initial condition. Consequently, we may use in Eq. (10) the closure approximation $\rho_{\mathrm{e}}^{2}=\kappa_{\mathrm{e}}^{2}$ and $\rho_{\mathrm{s}}^{2}=\rho_{0}^{2}$, while $\rho_{\mathrm{se}}^{2}$ and $\kappa_{\mathrm{s}}$ are zero at all times. We note that the same problems as in the present study were considered in two papers by Sedlacek [58, 59]. The results of Sedlacek can for all these problems be recovered from the present theory by setting $D=0$.

For the chosen geometry, $\kappa_{\mathrm{e}}$ is a function of the $x$ coordinate only. This leads to the equation

$$
\partial_{t} \kappa_{\mathrm{e}}=-\frac{\kappa_{\mathrm{e}}^{2}}{\rho^{2}} \partial_{x}(\rho v)+\rho \frac{\rho_{0}^{2}}{\rho^{2}} \partial_{x} v+\kappa_{\mathrm{e}} v k,
$$

where $\rho^{2}=\rho_{0}^{2}+\kappa_{\mathrm{e}}^{2}$. We further note that, for the plane-strain configurations under consideration, $\kappa_{\mathrm{e}}=-(1 / b) \partial_{x} \gamma$. In view of $\dot{\gamma}=\rho b v$ this leads to

$$
\frac{\rho_{0}^{2}}{\rho^{2}} \partial_{t} \kappa_{\mathrm{e}}=\rho \frac{\rho_{0}^{2}}{\rho^{2}} \partial_{x} v+\kappa_{\mathrm{e}} v k,
$$

The dynamic formulation is completed by specifying how the pseudo-scalar velocity $v$ relates to the local stress. As stated above, we use a linear stressvelocity relationship with a direction-independent dislocation mobility $B$. The local stress is considered as a sum of four contributions: (i) the mesoscopic stress $\tau(\boldsymbol{r})$ which derives from solving the elastic problem in a continuum setting using the strain field $\gamma(\boldsymbol{r})$. For the plane-strain geometries and initial conditions as specified above, this can be simply obtained from the density $\kappa_{\mathrm{e}}$, since $\kappa_{\mathrm{e}}=$ $-(1 / b) \partial_{x} \gamma$; (ii) the line-tension stress $T k / b$; (iii) the flow stress $\tau_{\mathrm{f}}$. In the present situation this is related to interactions with forest dislocations, as well as interactions between screw (but not edge) segments of opposite signs. Since both the density of screw dislocations and the planar density of forest dislocations intersecting the glide plane are conserved, $\tau_{\mathrm{f}}=\alpha G b \sqrt{\rho_{0}}$ is considered constant (a similar approximation of constant flow stress was made in $[58,59]$ ); (iv) the 
back stress $\tau_{\mathrm{b}}$, which from Eq. (7) is evaluated as

$$
\tau_{\mathrm{b}}=\frac{D G b}{\rho^{2}} \kappa_{\mathrm{e}} \partial_{x} \rho
$$

where we have used that the glide vectors of both dislocation orientations that may occur at a given point have the same $x$ component, $\boldsymbol{e}_{\mathrm{g}} \boldsymbol{e}_{x}=\kappa_{\mathrm{e}} / \rho$, and the system is homogeneous in the $y$ direction.

Since the flow stress has the character of a friction stress, whereas the other stresses are conservative in nature and may recover upon unloading, it is convenient to define the local stress as $\tau_{\text {loc }}=\tau(\boldsymbol{r})-\tau_{\mathrm{b}}-T k / b$. The dislocation velocity is then given by

$$
\frac{1}{b B} v=\left\{\begin{array}{l}
\tau_{\text {loc }}-\tau_{\mathrm{f}}, \quad \tau_{\text {loc }}>\tau_{\mathrm{f}}, \\
\tau_{\text {loc }}+\tau_{\mathrm{f}}, \quad \tau_{\text {loc }}<-\tau_{\mathrm{f}}, \\
0 \quad \text { otherwise }
\end{array}\right.
$$

In the following we focus on the quasistatic limit $B \rightarrow \infty$, which gives in general a good representation of the situation in weakly rate-dependent fcc crystals. In this case, we find that

$$
\begin{array}{lll}
k=\frac{\left(\tau(\boldsymbol{r})-\tau_{\mathrm{f}}\right) b}{T}-\frac{D G b^{2}}{T} \frac{\kappa_{\mathrm{e}}}{\rho^{2}} \partial_{x} \rho, & \tau_{\mathrm{loc}}>0, \\
k=\frac{\left(\tau(\boldsymbol{r})+\tau_{\mathrm{f}}\right) b}{T}-\frac{D G b^{2}}{T} \frac{\kappa_{\mathrm{e}}}{\rho^{2}} \partial_{x} \rho, & \tau_{\text {loc }}<0 .
\end{array}
$$

In the quasistatic limit, equation (14) reduces to a balance equation between segment multiplication and segment rotation terms:

$$
0=\frac{\rho_{0}^{2}}{\rho} \partial_{x} v+\kappa_{\mathrm{e}} v k
$$

where $v=$ const $/ \rho$. Combining Eqs. (17) and (18) we arrive at the differential equation

$$
\frac{\rho_{0}^{2}+D \kappa_{\mathrm{e}}^{2}}{\rho^{2}} \partial_{x} \rho=\frac{\kappa_{\mathrm{e}}}{G b}\left(\tau(\boldsymbol{r})-\tau_{\mathrm{f}}\right) .
$$

In the following, it is convenient to use non-dimensional variables defined by $\tilde{x}=x \sqrt{\rho_{0}}$ where $1 / \sqrt{\rho_{0}}$ is the characteristic length of the dislocation system, $\tilde{\kappa_{\mathrm{e}}}=\kappa_{\mathrm{e}} / \rho_{0}, \tilde{\rho}=\rho / \rho_{0}$, and $\tilde{\tau}=\tau / G b \sqrt{\rho_{0}}$. This gives the nondimensional equation

$$
\frac{1+D \tilde{\kappa}_{\mathrm{e}}^{2}}{\tilde{\rho}^{2}} \partial_{\tilde{x}} \tilde{\rho}=\tilde{\kappa_{\mathrm{e}}}(\tilde{\tau}(\boldsymbol{r})-\alpha) .
$$

From now on we will always refer to Equation (20) for describing the dislocation density pattern. Accordingly, in the following all variables are understood as scaled, and tildes are omitted.

\subsection{Shearing of a thin film on an elastic substrate}

As our first example, we envisage the shearing deformation of a thin film tethered to an elastic substrate. Using the continuum dislocation representation formulated above, we will examine the size-dependent deformation behavior of 
this system in both single and symmetrical double slip, and investigate similarities and differences with previous theoretical approaches.

In the following we adopt, instead of the slip system specific coordinate system used to formulate the model equations, a Cartesian coordinate system where the $x$ axis is normal to the plane of the film. We consider a homogeneous thin film of thickness $H$ with infinite extension in the $y$ and $z$ directions as depicted in Figure 1. The Burgers vector of the active slip system is contained in the $x z$ plane and forms an angle $\theta$ with the $x$ direction (two angles $\pm \theta$ in case of symmetrical double slip). The elastic boundary condition on the free surface of the film is $\sigma_{x x}(H)=0$, and a constant surface traction $\sigma_{x z}$ is applied to this surface. The dislocation densities must fulfil the boundary condition $\kappa_{\mathrm{e}}(H)=0$ [60]. (In fact, the problem under consideration is equivalent to the shear deformation of a constrained channel with elastic walls on both sides; the free surface of the thin film simply corresponds to the symmetry plane in the middle of the channel [60].) The requirement of zero dislocation flux through the interface with the elastic substrate $(\dot{\gamma}(0)=0$ for all times) imposes the boundary condition $\gamma(0)=0$ for the plastic strain. As initial condition we assume on each slip system a density $\rho_{0}$ of pure screw dislocations, with equal numbers of dislocations of both signs.

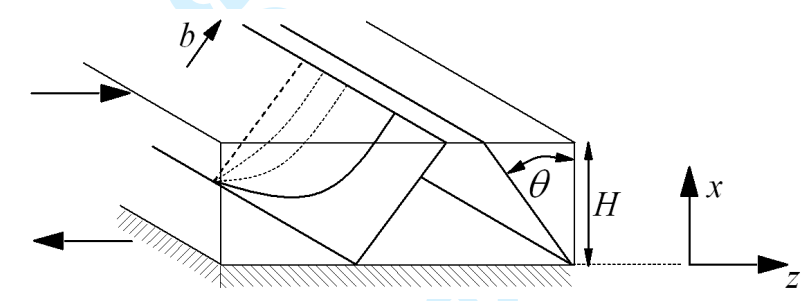

Figure 1: Illustration of the free surface thin film shearing case.

The whole problem can be easily identified as a plane strain one. Since all variables depend on the $x$ coordinate only , the formulation effectively becomes one-dimensional. The cases of single and symmetrical double slip are considered separately.

\subsubsection{Symmetrical double slip}

For deformation in symmetrical double slip, the resolved shear stress in each slip system is constant and given by $|\tau|=\tau_{\text {ext }}=-\sigma_{x z} \cos 2 \theta$ (see Appendix B). For each of the two slip systems, Eq.(20) transforms to

$$
\frac{\cos \theta\left(1+D \kappa_{\mathrm{e}}^{2}\right)}{\rho^{2}} \partial_{x} \rho=-\kappa_{\mathrm{e}}\left(\tau_{\mathrm{ext}}-\alpha\right),
$$

where $\rho=\sqrt{1+\kappa_{\mathrm{e}}^{2}}$ and the projection term $\cos \theta$ stems from the fact that the $x$ coordinate now refers to the film normal direction instead of the slip direction of the two slip systems. Solution of Eq.(20) is quite straightforward, namely

$$
x=H-\frac{\cos \theta}{\tau_{\mathrm{ext}}-\alpha}\left[(1-D) \frac{\kappa_{\mathrm{e}}}{\rho}+D \operatorname{arcsinh} \kappa_{\mathrm{e}}\right] .
$$

where $H$ is the scaled film height. 
In the following we measure $\gamma$ in units of $\left(b \sqrt{\rho_{0}}\right) / \cos \theta$ so that $\partial_{x} \gamma=-\kappa_{\mathrm{e}}$ in scaled variables. The relation between $\gamma$ and the dislocation density $\rho$ from substitution into Eq.(22) becomes

$$
\gamma=\frac{\cos \theta}{\tau_{\text {ext }}-\alpha}\left(\frac{(D-1)+D \rho^{2}}{\rho}\right)+c_{1},
$$

where the constant $c_{1}$ is determined from the boundary condition $\gamma(0)=0$.

Figure 3 shows the size-dependent hardening behavior observed in the cases $D=0$ (only line tension) and $D=1$ (line tension and back stress). In the former case, one observes an Orowan behavior with an initial transient (bowing out of dislocations) and then perfect plasticity at a critical stress which scales in inverse proportion with the film thickness. The behavior for $D=1$ is very different, as the system displays sustained hardening with a flow stress that increases, at large strains, approximately as the logarithm of strain.

To further elucidate this behavior, it is useful to have a look at the strain profiles shown in Figure 3. For $D=0$ these are segments of ellipses and, at the critical stress, $\partial_{x} \gamma$ diverges at the interface with the elastic substrate, indicating the onset of sustained dislocation motion at a constant stress level. (We note that for $D=0$ and at the critical stress, the boundary condition $\gamma=0$ at the interface need no longer be fulfilled. For $D>0$, on the other hand, $\gamma=0$ at the interface for all stresses). For $D=1$ the initial behavior is similar. At larger strains, however, a roughly flat strain profile develops and a boundary layer emerges at the film-substrate interface. The width of this layer is inversely proportional to the applied stress, and the strain and dislocation density profiles are roughly exponential. This can be seen immediately by noting that the boundary layer is characterized by a large density of dislocations in near-edge orientations such that $\left|\kappa_{\mathrm{e}}\right| \approx \rho \gg 1$ in Eq. (21). The density of edge dislocations piled up against the interface increases with increasing strain, and the ever increasing back stress of these dislocations leads to the observed hardening behavior.

\subsubsection{Single Slip}

In the case of single slip, the local stress is reduced by an amount $\tau_{\mathrm{mf}}=-\Theta \gamma$ which is proportional to the local strain (see Appendix). In non-dimensional variables, the coefficient $\Theta$ is given by $\Theta=(\sin 2 \theta)^{2} /(2(1-\nu) \cos \theta)$. Hence our governing equation is

$$
\frac{\cos \theta\left(1+D \kappa_{\mathrm{e}}^{2}\right)}{\rho^{2}} \partial_{x} \rho=\kappa_{\mathrm{e}}\left(\tau_{\mathrm{ext}}-\alpha-\Theta \gamma\right) .
$$

Differentiating with respect to $x$ and using $\partial_{x} \gamma=-\kappa_{\mathrm{e}}$ and $\rho^{2}=1+\kappa_{\mathrm{e}}^{2}$ yields

$$
\frac{1+D \kappa_{\mathrm{e}}^{2}}{\rho^{3}} \partial_{x x} \kappa_{\mathrm{e}}+\partial_{\kappa_{\mathrm{e}}}\left(\frac{1+D \kappa_{\mathrm{e}}^{2}}{\rho^{3}}\right)\left(\partial_{x} \kappa_{\mathrm{e}}\right)^{2}=(\Theta / \cos \theta) \kappa_{\mathrm{e}} .
$$

Using the substitution $w=\frac{1+D \kappa_{\mathrm{e}}^{2}}{\rho^{3}} \partial_{x} \kappa_{\mathrm{e}}$ into Eq.(25) we obtain an Abel equation of the form

$$
w \frac{\partial w}{\partial \kappa_{\mathrm{e}}}=\frac{\Theta}{\cos \theta} \kappa_{\mathrm{e}} \frac{1+D \kappa_{\mathrm{e}}^{2}}{\rho^{3}},
$$




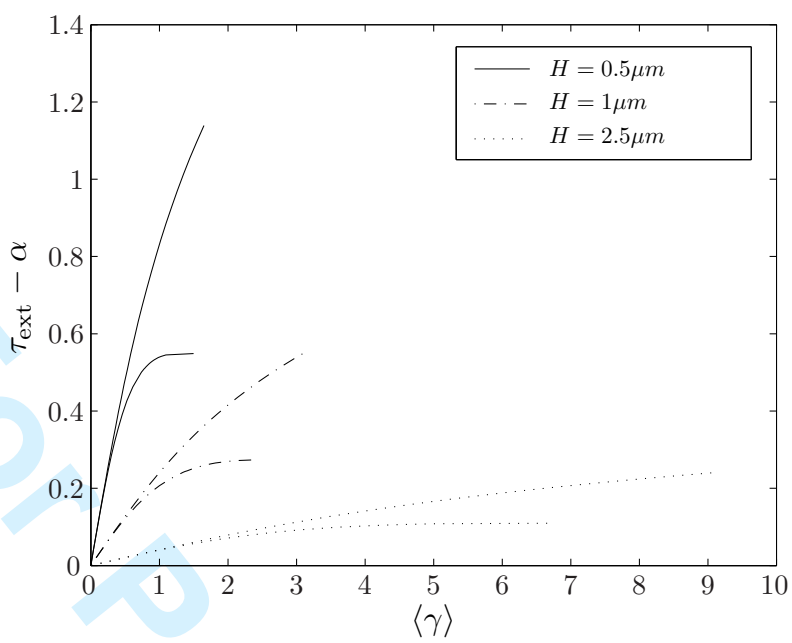

Figure 2: Stress-strain curves for films of different thickness deforming in symmetrical multiple slip; parameters: $\rho_{0}=10^{13} \mathrm{~m}^{2}, \theta=\pi / 6$, and $b=2.5 \cdot 10^{-10} \mathrm{~m}$. In each case the lower curve refers to $D=0$ and the upper curve to $D=1$.
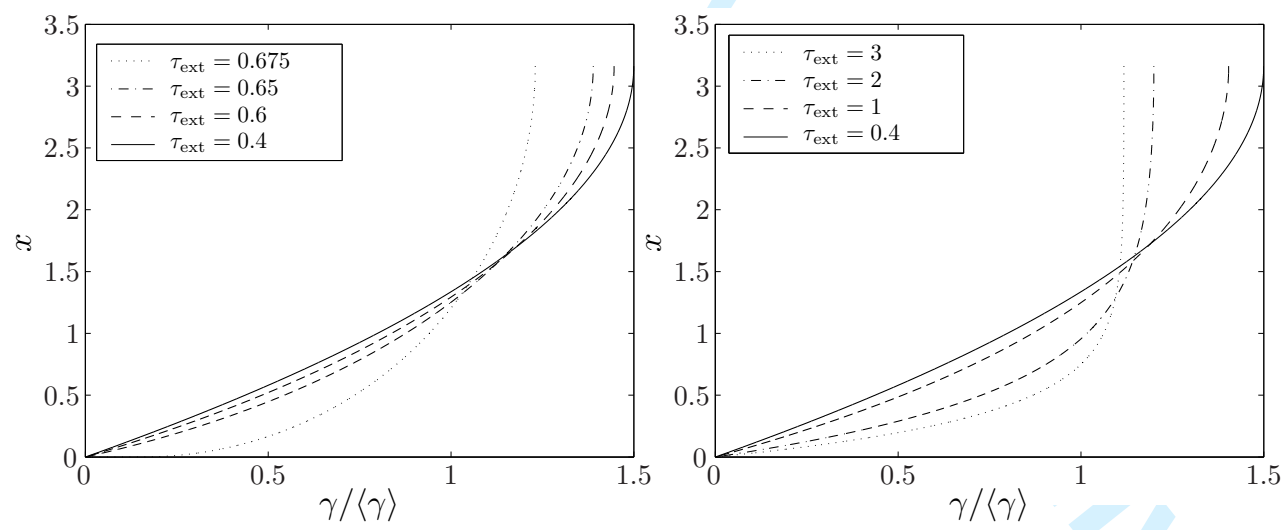

Figure 3: Plastic strain profiles for the cases $D=0$ (left) and $D=1$ (right), thin film shearing in symmetrical double slip. In both cases the film thickness is $H=1 \mu m$, and the other parameters are as in Figure. Stress values have been chosen to cover the full behaviour range from bowing initiation to advanced hardening. 


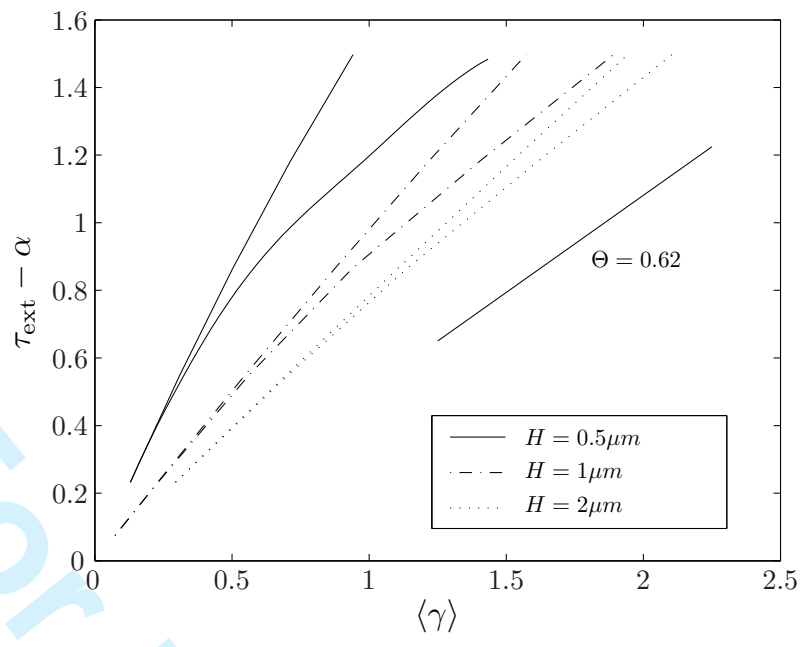

Figure 4: Stress-strain curves for films of different thickness deforming in single slip; parameters as in Figure 3. In each case the lower curve refers to $D=0$ and the upper curve to $D=1$.

from which we find that

$$
\partial_{x} \kappa_{\mathrm{e}}= \pm \frac{\rho^{3}}{1+D \kappa_{\mathrm{e}}^{2}} \sqrt{c+2 \frac{\Theta}{\cos \theta}\left(\frac{D-1}{\rho}+D \rho\right)} .
$$

In order to determine the value of the integration constant we adopt an iterative procedure since the imposed boundary conditions do not allow for direct evaluation. Starting from the condition $\kappa_{\mathrm{e}}(H)=0$ we seek $c$ values that eventually satisfy the prescribed relation $\gamma(0)=0$. Alternatively, a purely numerical solution based on a forward difference has been used and found to produce equally accurate and less cumbersome results.

The deformation behavior at first glance differs significantly from that in symmetrical double slip. The reason for this is that a mesoscopic 'fibre stress' is building up in the film.

This stress is proportional to the strain and counteracts the externally applied stress. As a consequence, one observes an apparent hardening even in the $D=0$ case. In this case, after an initial transient which depends on specimen size, an asymptotically linear stress-strain relationship with size-independent slope $\Theta$ is approached (see Figure 4). For $D=1$, on the other hand, the presence of an additional 'back stress' due to dislocations piling up against the film-substrate interface leads to an enhanced hardening which becomes more pronounced as the specimen size decreases.

The presence of the strain dependent 'fibre stress' diminishes the visible differences in strain profile between the $D=0$ and $D=1$ cases (Figure 5). However, the asymptotic characteristics of the profiles in the large-strain limit remain similar to the double slip case, viz, a semi-elliptical profile with vertical slope near the film-substrate interface for $D=0$, and two boundary layers of diminishing thickness for $D=1$. This indicates that, in spite of the apparent hardening associated with the build-up of a 'fibre stress' in the film during 
single-slip deformation, the basic deformation mechanisms in either case are the same as for symmetrical double slip.
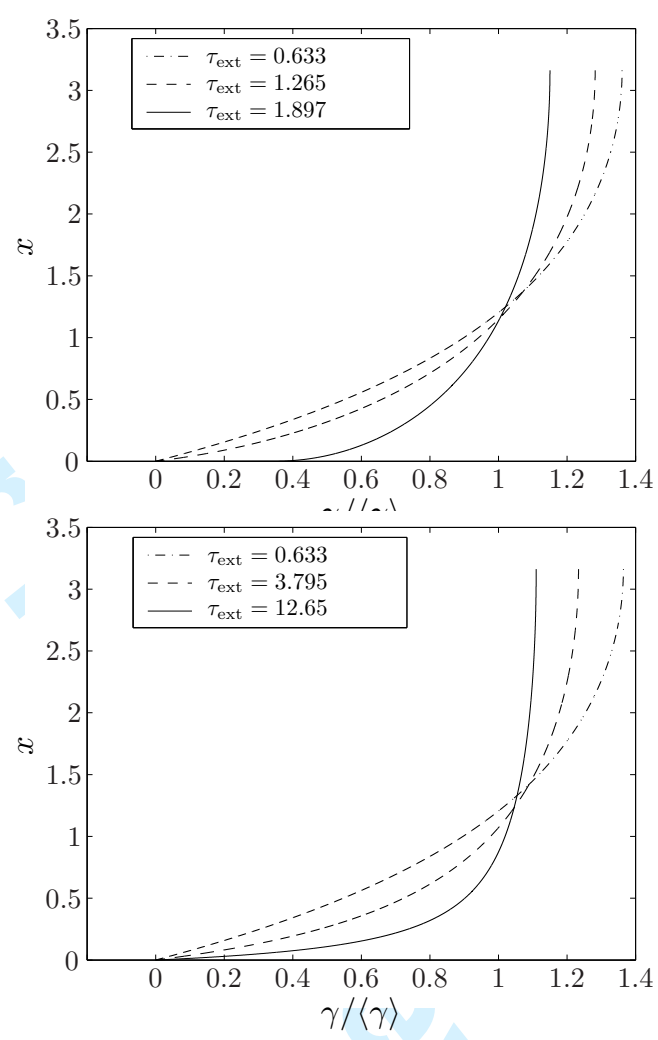

Figure 5: Plastic strain profiles for $D=0$ (left) and $D=1$ (right), thin film shearing in single slip. Parameters as in Figure 3.

\subsection{Bending of a free-standing thin film}

The next problem we consider concerns the bending deformation of a free standing thin film with two symmetrically inclined slip systems. The geometry is depicted in Figure 6, with a homogeneous film of infinite extension in the $y$ and $z$ directions and of thickness $2 H$ in the $x$ direction $(x \in[-H, H])$. At the free surfaces, we have the boundary conditions $\kappa_{\mathrm{e}}( \pm H)=0$ and, because of symmetry, $\gamma(0)=0$. It is therefore sufficient to solve the problem on the interval $x \in[0, H]$. We note in passing that Seldacek [59] treated the same problem by imposing that the strain be zero within an 'elastic core' of the strip. This assumption necessarily introduces discontinuities in the derivative of the strain $\gamma$, corresponding to points of infinite curvature of the dislocation lines. We find it difficult to see how this can be reconciled with the use of a line-tension approximation for describing the dislocation self-interactions.

The governing equation for this problem is similar to the previous ones, with the difference that the 'external' stress now is a linear function of the $x$ 
coordinate,

$$
\frac{\cos \theta\left(1+D \kappa_{\mathrm{e}}^{2}\right)}{\rho^{2}} \partial_{x} \rho=\kappa_{\mathrm{e}}(m x-\Theta \gamma-\alpha)=\kappa_{\mathrm{e}}\left(\tau_{\mathrm{ext}}(x)-\tau_{\mathrm{mf}}(\gamma, x)-\alpha\right) .
$$

where the constant $m$ depends on the applied bending moment (cf. below).

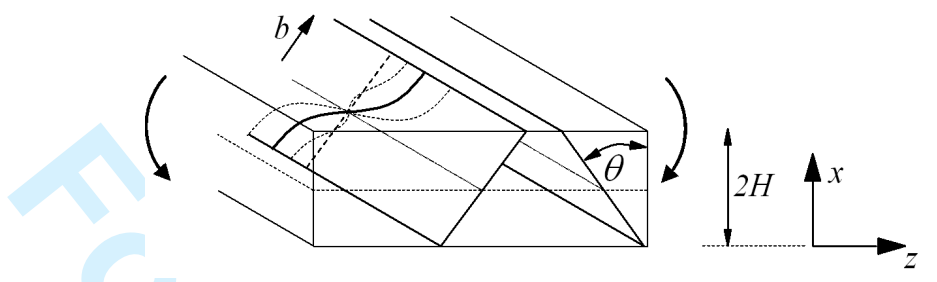

Figure 6: Illustration of the free standing thin film bending case.

Again, there is a mesoscopic internal stress in the film which is a function of the strain in either of the slip systems. Going through the same steps as in the previous sections, we finally obtain

$$
\frac{1+D \kappa_{\mathrm{e}}^{2}}{\rho^{3}} \partial_{x} \kappa_{\mathrm{e}}= \pm \sqrt{c+2 \frac{m\left[(1-D) \kappa_{\mathrm{e}}+D \rho \operatorname{arcsinh} \kappa_{\mathrm{e}}\right]+\Theta\left(D-1+D \rho^{2}\right)}{\rho \cos \theta}} .
$$

Again, we use an iterative procedure to obtain a solution which is consistent with the assumed boundary conditions. To this end, we chose a value of $m$ and then adjust the integration constant $c$ to satisfy the boundary condition $\kappa_{\mathrm{e}}=0$ at the free surface $x=H$. We then evaluate the strain profile, using $\partial_{x} \gamma=-\kappa_{\mathrm{e}}$ with the boundary condition $\gamma(0)=0$, and determine the average strain and the scaled bending moment $M$ defined by

$$
M=\frac{4}{\sin 2 \theta} \int_{0}^{H}\left(\tau_{\mathrm{ext}}+\tau_{\mathrm{mf}}\right) x d x=\frac{4}{\sin 2 \theta} \int_{0}^{H}(m x-\Theta \gamma) x d x .
$$

An investigation of strain profiles obtained in this manner (Figure 7) demonstrates significant differences with the shearing problem. Strain profiles for $D=0$ and $D=1$ are very similar, with strain fields that go smoothly to zero at $x=0$. No boundary layer effects are observed, and there is no evidence of an 'elastic core' as imposed by Seldacek [59] through a corresponding boundary condition. A comparison with the results of Sedlacek shows that, in the present model, strains may become negative in the vicinity of the neutral axis, i.e., dislocations there bow in a direction opposite to that imposed by the applied bending moment. (An explicit comparison of strain profiles obtained with our and with Sedlacek's boundary condition has been given by Sedlacek in the appendix to [59].) Generally speaking, our theory yields larger strains for small stresses (the system is softer), however, for large bending moments our $D=0$ results approach those of Sedlacek, since in this limit the boundary conditions become asymptotically similar (zero strain at or near $x=0$ ).

In spite of the apparent similarity in the strain profiles the hardening behavior in the $D=0$ and $D=1$ cases is quite different. This can bee seen in Figure 

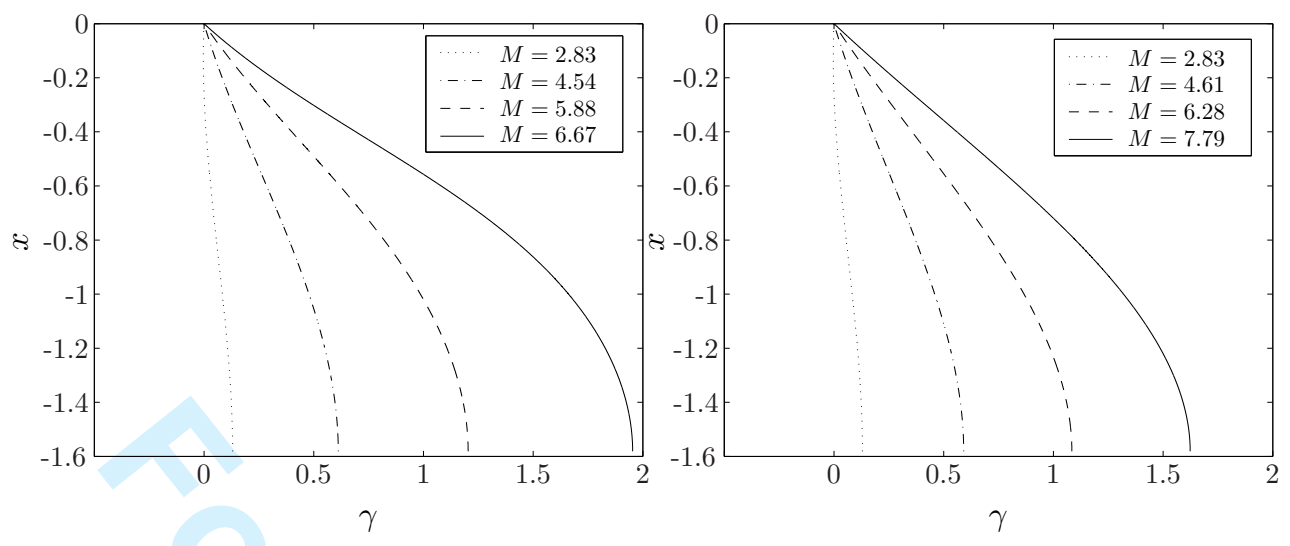

Figure 7: Inhomogeneous plastic strain distribution in bending for $D=0$ (left) and $D=1$ (right). Both figures are produced for film thickness $2 H=1 \mu \mathrm{m}$, other parameters as in Figure 3

8 which shows for different film thicknesses $H$ plots of the normalized bending moment $M / H^{2}$ versus the average strain in a half-strip, $\{\gamma\}=\int_{0}^{H} \gamma(x) \mathrm{d} x$.

In the absence of the 'back stress' term $(D=0)$, the normalized bending moment $M / H^{2}$ approaches a constant value which for large $H$ is proportional to the flow stress constant $\alpha$. For smaller $H$, one observes an initial transient and an asymptotically constant level of $M / H^{2}$ which is roughly in inverse proportion with $H$, indicating an Orowan-type size effect. If the back stress term is taken into account $(D=1)$, on the other hand, the deformation behavior changes significantly. Owing to the condition $\gamma(0)=0$, dislocations increasingly bend into edge orientations and the accumulation of edge dislocations of the same sign in either half-strip leads to an ever increasing back stress which gives rise to sustained hardening, with a hardening rate that increases strongly with decreasing film thickness.

\section{Conclusions}

We have presented a framework which generalizes previous approaches towards density-based dislocation dynamics in order to describe the kinematics and interactions of curved dislocation lines. In conclusion of this study, it is useful to discuss the relationship of the present approach with other density-based approaches. In doing so, we shall focus on problems related to the formulation of statistically averages and the incorporation of 'microscopic' dislocation interactions into such theories. A mathematical discussion of the formal relations between different density-based approaches that have been proposed for describing systems of curved dislocations [20, 48, 49] can be found elsewhere $[50,51]$.

The different theories proposed in the literature differ in the density measures used. Accordingly, the evolution equations have at first glance different formal structure. For systems of discrete dislocation lines, however, any sensible theory reduces to the classical kinematic evolution equation of the dislocation 

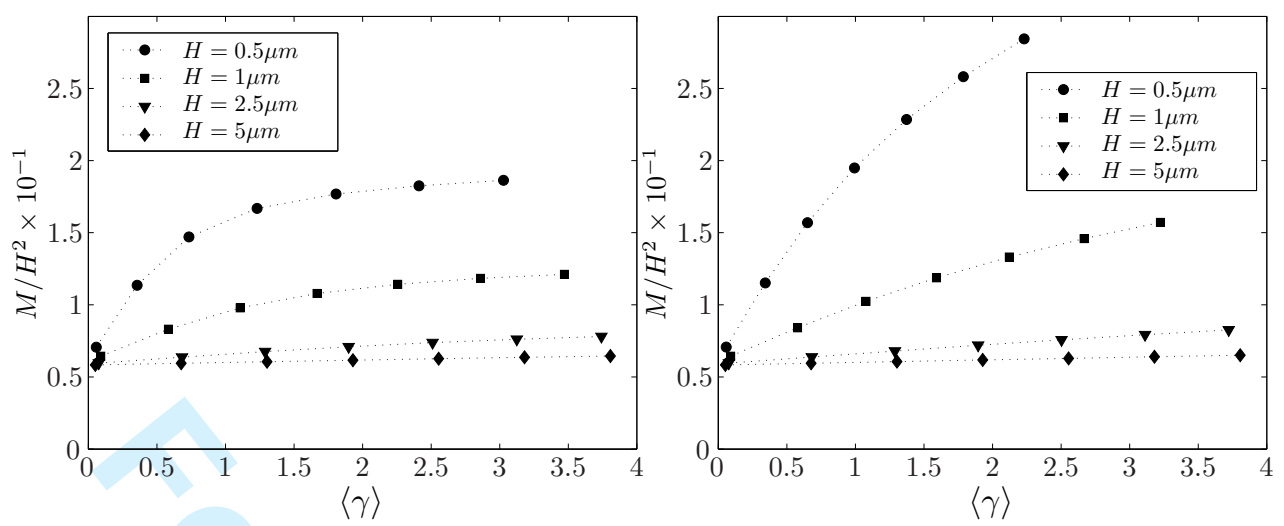

Figure 8: Size dependence of the bending response (Strain average over halfstrip vs. normalized bending moment) for $D=0$ (left) and $D=1$ (right). Parameters as in previous figures.

density tensor, and therefore for this case all density-based theories tend to be equivalent. The same is true for continuously distributed bundles of parallel dislocations, where it is possible to uniquely assign a dislocation orientation to each point in space. As far as the kinematics of such problems is concerned, the choice of any particular theory seems to be largely a matter of taste.

Problems arise, however, as soon as one leaves the discrete-dislocation scale and proceeds to do averages. In general, dislocation arrangements consist of segments of multiple orientations within one and the same mesoscopic volume element. This leads to two problems: (i) Relevant kinematic information is lost if one uses the average dislocation density tensor only. This problem is well-known: The rotation-free plastic distortion which results from the motion of 'statistically stored' dislocations with zero net Burgers vector cannot be captured in terms of the averaged dislocation density tensor, which depends only on the excess Burgers vector contained within the averaging volume. (ii) A second problem is less commonly addressed. The dynamics of dislocation segments depends on the stresses acting on the segments. It is therefore inadequate to describe the local stress state in terms of a simple volume average over some mesoscopic volume - rather, one has to take conditional averages over only those positions within the meoscopic volume element which are actually occupied by the type of dislocations under consideration. Speaking in physical terms, one has to find a way of keeping track of the relative orientation of dislocation segments and the topological structure of the dislocation network, as these are the parameters which determine the local flow stress.

In the present paper, we used a semi-phenomenological model which describes 'microscopic' dislocation interactions in terms of a local flow stress fulfilling Taylor's relation, plus two non-local terms related to dislocation curvature ('line tension' stress) and to repulsive stresses between individual dislocations in pile-up configurations ('back stress'). In particular, we have shown for different constrained deformation geometries (shearing of a thin film (or equivalently a slip channel), microbending) that the first of these terms leads to an Orowantype size effect on the flow stress. Inclusion of the second term leads to sustained 
size-dependent hardening as dislocations in near-edge orientations with increasing strain pile up against the intrinsic boundaries (the film-substrate interface in case of thin film shearing and the neutral fiber in the case of bending). In the thin film case, the 'back stress' term gives rise to the formation of a boundary layer with an excess dislocation density that decreases exponentially from the boundary, and a corresponding increase of the strain. Similar exponential boundary layers have been reported in experiments (deformation of bicrystals [61]) as well as in discrete simulations of deformation of thin films or, equivalently, slip channels [62].

Further investigations are required to validate the present or similar continuum approaches by comparing the results with discrete dislocation dynamics simulations. The present theory is capable of qualitatively reproducing some key features of discrete simulations which have been proposed as benchmarks for size-dependent plasticity [46, 62]. It allows to understand size effects on flow stress and hardening, as well as the formation of boundary layers in constrained plasticity. However, a quantitative comparison between continuum theory and discrete simulation is not yet possible since the basic assumptions are too different: None of the presently available continuum models has yet managed to include in a consistent manner the operation of localized sources (as opposed to the bowing of dislocations which thread the entire system). On the other hand, the simulations which have been proposed as benchmarks in the literature are two-dimensional (systems of straight parallel dislocations) and can therefore not account for the effects of line tension and dislocation bowing. In the next step, we think it is therefore necessary to include dislocation multiplication into the present, or related, continuum theories and to provide 3D benchmark simulations for assessing the validity of such generalizations.

From a continuum mechanics viewpoint it is interesting to note that both the 'back stress' and 'line tension' terms can be understood as stresses which relate to second-order gradients of the plastic strain: The line tension is a second gradient of the plastic shear strain in the dislocation line direction, and the back stress (or 'pile-up stress', cf. [43]) is a second gradient of the plastic shear strain in the direction normal to the dislocation line. This suggests that, in the spirit of Aifantis' proposition to introduce second-order strain gradients into macroscopic plastic flow rules (see e.g. $[33,39]$ ), it might be possible to unify both terms to yield a generic formalism of gradient-dependent dislocation dynamics. This is another issue to be explored in the future.

\section{Acknowledgments}

Acknowledgements: We acknowledge support of the Commission of the European Communities under contracts MRTN-CT-2003-506434 (SizeDepEn) and HPRN-CT 2002-00198, and of EPSRC under Grant No. GR/S20406/01.

\section{A Derivation of the edge-screw model}

We start out from the fundamental kinetic equation (1),

$$
\partial_{t} \rho=-\partial_{\phi}\left[\rho v_{\phi}\right]-\nabla \cdot[\rho \boldsymbol{v}]+\rho v k
$$


Note that, for a discrete dislocation system, the density function $\rho$ has the structure $\rho=\rho(\boldsymbol{r}) \delta(\phi-\phi(\boldsymbol{r}))$ since the dislocation orientation is uniquely defined in each point. The partial densities $R_{\mathrm{S}}$ and $R_{\mathrm{e}}$ are given by

$$
R_{\mathrm{s}}(\boldsymbol{r})=\int \rho \cos \phi \mathrm{d} \phi \quad, \quad R_{\mathrm{e}}(\boldsymbol{r})=\int \rho \sin \phi \mathrm{d} \phi .
$$

and for a discrete system the local dislocation orientation can be expressed through the relation $\tan \phi=R_{\mathrm{S}}(\boldsymbol{r}) / R_{\mathrm{e}}(\boldsymbol{r})=R_{\mathrm{S}} / R_{\mathrm{e}}$. Inserting these relations into the kinetic equation, and assuming an orientation-independent dislocation mobility (no explicit dependence of $|v|$ on $\phi$ ), we find

$$
\begin{aligned}
& \partial_{t} R_{\mathrm{s}}=R_{\mathrm{s}} v k-R_{\mathrm{e}}\left(\boldsymbol{e}_{\mathrm{t}} \nabla\right) v-\frac{R_{\mathrm{s}}}{\rho}\left(\boldsymbol{e}_{\mathrm{g}} \nabla\right)[\rho v], \\
& \partial_{t} R_{\mathrm{e}}=R_{\mathrm{e}} v k+R_{\mathrm{s}}\left(\boldsymbol{e}_{\mathrm{t}} \nabla\right) v-\frac{R_{\mathrm{e}}}{\rho}\left(\boldsymbol{e}_{\mathrm{g}} \nabla\right)[\rho v] .
\end{aligned}
$$

Finally we use that the dislocation glide and tangent vectors are given by $\boldsymbol{e}_{\mathrm{g}}=$ $\sin \phi \boldsymbol{e}_{x}-\cos \phi \boldsymbol{e}_{y}=\left(R_{\mathrm{e}} / \rho\right) \boldsymbol{e}_{x}-\left(R_{\mathrm{s}} / \rho\right) \boldsymbol{e}_{y}$ and $\boldsymbol{e}_{\mathrm{t}}=\cos \phi \boldsymbol{e}_{x}+\sin \phi \boldsymbol{e}_{y}=\left(R_{\mathrm{s}} / \rho\right) \boldsymbol{e}_{x}+$ $\left(R_{\mathrm{e}} / \rho\right) \boldsymbol{e}_{y}$, respectively. This leads to

$$
\begin{aligned}
& \partial_{t} R_{\mathrm{e}}=\left[-\frac{R_{\mathrm{e}}^{2}}{\rho^{2}} \partial_{x}(\rho v)+\frac{R_{\mathrm{e}} R_{\mathrm{s}}}{\rho^{2}} \partial_{y}(\rho v)\right]+R_{\mathrm{s}}\left[\frac{R_{\mathrm{s}}}{\rho} \partial_{x} v+\frac{R_{\mathrm{e}}}{\rho} \partial_{y} v\right]+R_{\mathrm{e}} v k, \\
& \partial_{t} R_{\mathrm{s}}=\left[-\frac{R_{\mathrm{e}} R_{\mathrm{s}}}{\rho^{2}} \partial_{x}(\rho v)+\frac{R_{\mathrm{s}}^{2}}{\rho^{2}} \partial_{y}(\rho v)\right]-R_{\mathrm{e}}\left[\frac{R_{\mathrm{s}}}{\rho} \partial_{x} v+\frac{R_{\mathrm{e}}}{\rho} \partial_{y} v\right]+R_{\mathrm{s}} v k .
\end{aligned}
$$

When averaging these equations, it is necessary to consider terms of the type $\left\langle R_{\mathrm{s}}^{2} / \rho^{2}\right\rangle$ or $\left\langle R_{\mathrm{s}} / \rho\right\rangle$. The average is understood as an average over some mesoscopic volume $\Delta V$ and all orientations of segments within that volume. To prove some useful relationships, we divide all dislocation lines contained within $\Delta V$ into short segments of length $l$, which we label with the discrete index $\beta$. The edge and screw lengths of a given segment are $l_{\mathrm{s}}^{\beta}=l \cos \phi_{\beta}$ and $l_{\mathrm{e}}^{\beta}$. Clearly, $\rho=N_{V} l / V$ where $N_{V}$ is the number of segments in $V$, and $\kappa_{\mathrm{s}, \mathrm{e}}:=\left\langle R_{\mathrm{s}, \mathrm{e}}\right\rangle=\sum_{\beta \in V} l_{\mathrm{s}, \mathrm{e}}^{\beta} / V$. It is then easy to see the identities

$$
\begin{array}{r}
\left\langle\frac{R_{\mathrm{e}}^{2}}{\rho^{2}}\right\rangle=\frac{1}{V} \sum_{\beta \in V} \frac{\left(l_{\mathrm{e}}^{\beta}\right)^{2}}{l}=\frac{1}{N_{V}} \sum_{\beta \in V} \frac{\left(l_{\mathrm{e}}^{\beta}\right)^{2}}{l^{2}} \frac{N_{V}}{l} V=\frac{\left\langle R_{\mathrm{e}}^{2}\right\rangle}{\left\langle\rho^{2}\right\rangle}, \\
\left\langle\frac{R_{\mathrm{e}}^{2}}{\rho}\right\rangle=\frac{1}{N_{V}} \sum_{\beta \in V} \frac{\left(l_{\mathrm{e}}^{\beta}\right)^{2}}{l^{2}}=\frac{\sum_{\beta \in V}\left(l_{\mathrm{e}}^{\beta}\right)^{2}}{\sum_{\beta \in V} l^{2}}=\frac{\left\langle R_{\mathrm{e}}^{2}\right\rangle}{\left\langle\rho^{2}\right\rangle}\langle\rho\rangle,
\end{array}
$$

and similarly for $R_{\mathrm{s}}$.

\section{B Elastic solutions}

To find the elastic solutions required in the examples we assume an isotropic linear-elastic solid and use a small-strain approximation. The stress is related to the elastic and plastic strain tensors by $\boldsymbol{\sigma}=\boldsymbol{E}\left(\varepsilon^{\mathrm{tot}}-\varepsilon^{\mathrm{pl}}\right)$. The total strain tensor $\boldsymbol{\varepsilon}^{\text {tot }}$ is the symmetric part of the elastic distortion tensor $\boldsymbol{\beta}=\operatorname{Grad} \boldsymbol{u}$ 
where $\boldsymbol{u}=\left[u_{x}, u_{y}, u_{z}\right]$ is the displacement field, and $\boldsymbol{\varepsilon}^{\mathrm{pl}}$ is the symmetric part of the plastic distortion tensor $\boldsymbol{\beta}^{\mathrm{pl}}$. The latter is determined from the crystal plasticity relation $\boldsymbol{\beta}^{\mathrm{pl}}=\sum_{i} \gamma_{i} \boldsymbol{e}_{\mathrm{g}}^{i} \otimes \boldsymbol{n}^{i}$ (the sum runs over all active slip systems).

Using the coordinate system in Figures 1 and 6, we find that the slip and slip plane normal vectors in the case of symmetrical double slip are given by

$$
\begin{aligned}
& \boldsymbol{e}_{\mathrm{g}}^{1}=\left[\begin{array}{lll}
\cos \theta & 0 & \sin \theta
\end{array}\right], \quad \boldsymbol{e}_{\mathrm{g}}^{2}=\left[\begin{array}{lll}
\cos \theta & 0 & -\sin \theta
\end{array}\right], \\
& \boldsymbol{n}^{1}=\left[\begin{array}{lll}
\sin \theta & 0 & -\cos \theta
\end{array}\right], \quad \boldsymbol{n}^{2}=\left[\begin{array}{lll}
\sin \theta & 0 & \cos \theta 37)
\end{array}\right.
\end{aligned}
$$

and the elastic strain tensor follows as

$$
\left(\varepsilon^{\mathrm{tot}}-\varepsilon^{\mathrm{pl}}\right)=\frac{1}{2}\left[\begin{array}{ccc}
2 u_{x}^{\prime}-\left(\gamma_{1}+\gamma_{2}\right) \sin 2 \theta & 0 & u_{z}^{\prime}+\left(\gamma_{1}-\gamma_{2}\right) \cos 2 \theta \\
0 & 0 & 0 \\
u_{z}^{\prime}+\left(\gamma_{1}-\gamma_{2}\right) \cos 2 \theta & 0 & \left(\gamma_{1}+\gamma_{2}\right) \sin 2 \theta
\end{array}\right]
$$

The prime denotes derivative with respect to $x$ coordinate (all other derivatives vanish). The resolved shear stresses for each slip system are obtained via $\tau^{i}(\boldsymbol{r})=$ $\boldsymbol{n}^{i} \cdot\left[\boldsymbol{\sigma} e_{\mathrm{g}}^{i}\right]$, and the case of single slip is simply recovered by setting $\gamma_{2}=0$.

Due to the superposition principle, the stress state can then be considered as a superposition of a 'mesoscopic' internal stress related to the shear strain fields $\gamma_{1}$ and $\gamma_{2}$, which is evaluated for a body with traction-free surfaces, and an 'external stress' which corresponds to the stress state of the same body under the prescribed boundary conditions but without plastic distortions. Both fields separately fulfill the stress equilibrium condition $\operatorname{div} \sigma=0$. We first determine the internal stresses. The shear stress for each slip plane for both of the states discussed is given by

$$
\begin{aligned}
\tau^{1} & =\frac{\sigma_{x x}-\sigma_{z z}}{2} \sin 2 \theta-\sigma_{x z} \cos 2 \theta, \\
\tau^{2} & =\frac{\sigma_{x x}-\sigma_{z z}}{2} \sin 2 \theta+\sigma_{x z} \cos 2 \theta .
\end{aligned}
$$

Use of equilibrium conditions $\partial_{x} \sigma_{x z}=0, \partial_{x} \sigma_{x x}=0$ together with the tractionfree requirement provide us with $\sigma_{x z}=0$ and $\sigma_{x x}=0$. Thus for shear deformation in single slip the shear stress reduces to $\tau=-\sigma_{z z} \sin 2 \theta / 2$. By replacements in Hooke's law

$$
\begin{aligned}
\sigma_{x x} & =\frac{2 G(1-\nu)}{(1-2 \nu)}\left(\varepsilon_{x x}+\frac{\nu}{(1-\nu)} \varepsilon_{z z}\right) \\
\sigma_{z z} & =\frac{2 G(1-\nu)}{(1-2 \nu)}\left(\varepsilon_{z z}+\frac{\nu}{(1-\nu)} \varepsilon_{x x}\right), \\
\sigma_{x z} & =2 G \varepsilon_{x z}
\end{aligned}
$$

we eventually obtain

$$
\tau_{\mathrm{mf}}=-\frac{\sin ^{2} 2 \theta}{2(1-\nu)} G \gamma_{1}
$$

For shear deformation in symmetrical double slip, $\gamma_{1}=-\gamma_{2}$ and, hence, $\tau_{\mathrm{mf}}=0$. For bending in symmetrical double slip, on the other hand, $\gamma_{1}=\gamma_{2}=: \gamma / 2$, and we similarly find for either of the slip systems

$$
\tau_{\mathrm{mf}}=-\frac{\sin ^{2} 2 \theta}{2(1-\nu)} G \gamma .
$$


In the most general case of arbitrary deformations on two generically inclined slip systems, finally, one obtains

$$
\begin{gathered}
\left.\tau_{\mathrm{mf}}^{1}=-G \frac{\gamma_{1} \sin ^{2} 2 \theta_{1}+\gamma_{2} \sin 2 \theta_{1} \sin 2 \theta_{2}}{2(1-\nu)}\right), \\
\tau_{\mathrm{mf}}^{2}=-G \frac{\gamma_{2} \sin ^{2} 2 \theta_{2}+\gamma_{1} \sin 2 \theta_{1} \sin 2 \theta_{2}}{2(1-\nu)} .
\end{gathered}
$$

In this case, two coupled equations for the evolution of strain on the two slip systems have to be considered.

Stresses due to externally applied tractions superimpose on the internal stresses evaluated above. For shearing, $\partial_{x} \sigma_{x z}=0, \partial_{x} \sigma_{x x}=0$ together with the prescribed tractions simply yield a constant external stress

$$
\tau_{\mathrm{ext}}=-\sigma_{x z} \cos 2 \theta .
$$

For bending assuming a constant curvature with bending radius $R>P$, having only $\sigma_{z z}$ to be non zero in the plane, one obtains a linear stress profile

$$
\tau_{\mathrm{ext}}=-\frac{G \sin 2 \theta}{1-\nu} \frac{x}{R}
$$

\section{References}

[1] Dislocations in Solids: On dislocation patterning, edited by H. Suzuki, T. Ninomiya, K. Sumino, S. Takeuchi, (Tokyo Univ. Press, 1985).

[2] E. C. Aifantis, Mater. Sci. Eng. 81563 (1986).

[3] B. Devincre and M. Condat, Acta Metall. Mater. 402629 (1992).

[4] B. Devincre and L. P. Kubin, Mater. Sci. Eng. A 8234 (1997).

[5] N. M. Ghoniem, S. H. Tong and L. Z. Sun, Phys. Rev. B 61913 (2000).

[6] H. M. Zbib, T. D. de La Rubia, R. Rhee and J. P. Hirth, J. Nucl. Mater. 276154 (2000).

[7] V. Bulatov, J. F. Justo, W. Cai, et al., Phil. Mag. A 811257 (2001).

[8] Y. U. Wang, Y. M. Jin, A. M. Cuitino and A. G. Khachaturyan, Acta Mater. 491847 (2001).

[9] M. Koslowski, A. M. Cuitino and M. Ortiz, J. Mech. Phys. Solids 502597 (2002).

[10] A. Acharya, Journal Mech. Phys. Solids 49761 (2001).

[11] A. Acharya, this volume.

[12] U. F. Kocks, J. Eng. Mater. Technol. 9876 (1976).

[13] L. P. Kubin and Y. Estrin, Rev. Phys. Appl. 23573 (1988).

[14] D. Kuhlmann-Wilsdorf and J. H. Van Der Merwe, Mater. Sci. Eng. 5579 (1982). 
[15] L. Holt, J. Appl. Phys. 413197 (1970).

[16] K. Kondo, in Proc. 2. Japan Nat. Congress of Appl. Mech. (1952).

[17] J. F. Nye, Acta Metallurgica 1152 (1953).

[18] E. Kröner, Kontinuumstheorie der Versetzungen und Eigenspannungen (Springer-Verlag, Berlin, 1958).

[19] T. Mura, Phil. Mag. 8843 (1963).

[20] A. M. Kosevich, in Dislocations in Solids, Vol. 1, (North Holland, Amsterdam, 1979), pp. 33-142.

[21] E. Kröner, Continuum theory of defects (North Holland, Amsterdam, 1981).

[22] E. C. Aifantis, in Defects Fracture and Fatigue: Dislocation kinetics and the formation of deformation bands, edited by G. C. Sih and J. W. Provan, (Martinus-Nijhoff, The Hague, 1981), pp. 75-84.

[23] D. J. Bammann and E. C. Aifantis, Acta Mechanica 4591 (1982).

[24] D. Walgraef and E. C. Aifantis, J. Appl. Phys. 58688 (1985).

[25] D. Walgraef and E. C. Aifantis, Res Mechanica 23161 (1988).

[26] J. Pontes, D. Walgraef and E. C. Aifantis, Int. J. Plasticity 221486 (2006).

[27] A. E. Romanov and E. C. Aifantis, Scripta Met. Mat. 29707 (1993).

[28] A. E. Romanov and E. C. Aifantis, Scripta Met. Mat. 30 1293, 1581 (1994).

[29] J. Kratochvil and S. Libovicky, Scripta Metall. 201625 (1986).

[30] J. Kratochvil and R. Sedlacek, Phys. Rev. B 67094105 (2003)

[31] E. C. Aifantis, J. Eng. Mater. Tech. 121189 (1999).

[32] E. C. Aifantis, Int. J. Plasticity 3211 (1987).

[33] H. M. Zbib and E. C. Aifantis, Res Mechanica 23 261, 279, 293 (1988).

[34] P. Hähner, Mater. Sci. Eng. A 164, 23 (1993).

[35] N. A. Fleck, G. M. Muller, M. F. Ashby and J. W. Hutchinson, Acta Metall. 42475 (1994).

[36] W. D. Nix and H. Gao, J. Mech. Phys. Solids 46411 (1998).

[37] H. Gao, Y. Huang, W. D. Nix, and J. W. Hutchinson, J. Mech. Phys. Solids 471239 (1999).

[38] E. C. Aifantis, Int. J. Fracture 95299 (1999).

[39] E. C. Aifantis, Mechanics of Materials 35259 (2003).

[40] I. Tsagrakis, A. Konstantinides and E. C. Aifantis, Mechanics of Materials 35733 (2003). 
[41] A. Needleman and J. Gil Sevillano, Scripta Materialia 48109 (2002).

[42] M. Zaiser and E. C. Aifantis, Scripta Materialia 48133 (2002).

[43] I. Groma, F. Csikor and M. Zaiser, Acta Mater. 511271 (2003).

[44] M. Zaiser, M.-C. Miguel, and I. Groma, Phys. Rev. B 64224102 (2001).

[45] I. Groma, G. Györgyi and B. Kocsis, Phil. Mag (this volume).

[46] S. Yefimov, I. Groma and E. Van der Giessen, J. Mech. Phys. Solids 52 279 (2004).

[47] S. Yefimov, I. Groma and E. Van der Giessen, Modelling Simul. Mater. Sci. Eng. 121069 (2004).

[48] A. El-Azab, Phys. Rev. B 6111956 (2000).

[49] R. Sedlacek, J. Kratochvil, and E. Werner, Phil. Mag. 83, 3735 (2003).

[50] T. Hochrainer, M. Zaiser and P. Gumbsch, Phil. Mag. (this volume).

[51] T. Hochrainer and M. Zaiser, in Proc. International Conference on Statistical Mechanics of Plasticity and Related Instabilities: Fundamentals of a continuum theory of dislocations, PoS(SMPRI2005)002, (2006).

[52] M. Zaiser and T. Hochrainer, Scripta Mater. 54717 (2006).

[53] M.-C. Miguel, A. Vespignani, M. Zaiser and S. Zapperi, Phys. Rev. Letters 89165501 (2002).

[54] M. Zaiser and A. El-Azab, in Multiscale Phenomena in Materials, edited by H. M. Zbib, D. H. Lassila, L. E. Levine and K. J. Hemker, MRS Proceedings, Vol. 779, (MRS, Warrendale (PA), 2004), W5.7.

[55] A. El-Azab, J. Deng and M. Tang, this volume.

[56] J. Kratochvil, in Proc. 2nd Int. Conf. on Multiscale Materials Modelling, Ed. N.M. Ghoniem, (UCLA 2004), p. 222-224.

[57] A. Arsenlis, D. M. Parks, R. Becker, V. V. Bulatov, J. Mech. Phys. Solids 521213 (2004).

[58] R. Sedlacek and E. Werner, Phys. Rev. B 69134114 (2004)

[59] R. Sedlacek, Mater. Sci. Eng. A 393387 (2005).

[60] W. D. Nix, Metallurgical Transactions 202217 (1989).

[61] S. Sun, B.L. Adams and W.E. King, Philos. Mag. A 80, 9 (2000)

[62] J.Y. Shu, N.A. Fleck, E. Van der Giessen and A. Needleman, J. Mech. Phys. Solids 491361 (2001). 